\title{
CT Angiography ASPECTS Predicts Outcome Much Better Than Noncontrast CT in Patients with Stroke Treated Endovascularly
}

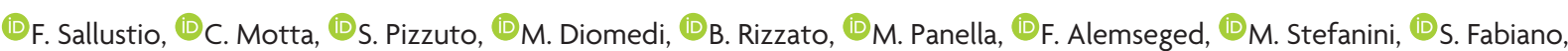
(1) R. Gandini, 1 R. Floris, DP. Stanzione, and (1) G. Koch

\begin{abstract}
BACKGROUND AND PURPOSE: Noncontrast CT ASPECTS has been investigated as a predictor of outcome in patients with acute ischemic stroke. Our purpose was to investigate whether CTA source images are a better predictor of clinical and radiologic outcomes than NCCT ASPECTS in candidates for endovascular stroke therapy.
\end{abstract}

MATERIALS AND METHODS: CT scans of patients $(n=124)$ were independently evaluated by 2 readers for baseline NCCT and CTA source image ASPECTS and for follow-up ASPECTS. An mRS of $\leq 2$ at 3 months was considered a favorable outcome. Receiver operating characteristic curve analysis was used to assess the ability of NCCT and CTA source image ASPECTS to identify patients with favorable outcomes. A stepwise multiple regression analysis was performed to find independent predictors of outcome.

RESULTS: Baseline CTA source image ASPECTS correlated better than NCCT ASPECTS with follow-up ASPECTS $(r=0.76$ versus $r=0.51$; $P$ for comparison of the 2 coefficients $<.001)$. Receiver operating characteristic curve analysis showed that baseline CTA source image ASPECTS compared with NCCT ASPECTS can better identify patients with favorable outcome (CTA source image area under the curve = $0.83 ; 95 \% \mathrm{Cl}, 0.76-0.91 ; \mathrm{NCCT}$ area under the curve $=0.67 ; 95 \% \mathrm{Cl}, 0.58-0.77 ; P<.001)$. Finally, the stepwise regression analysis showed that lower age, good recanalization, lower time to recanalization, and good baseline CTA source image ASPECTS, not NCCT ASPECTS, were independent predictors of favorable outcome.

CONCLUSIONS: CTA source image ASPECTS predicts outcome better than NCCT ASPECTS; this finding suggests CTA rather than NCCT as a main step in the decision-making process for patients with acute ischemic stroke.

ABBREVIATIONS: $\mathrm{CTA}-\mathrm{SI}=\mathrm{CTA}$ source images; $\mathrm{ET}=$ endovascular stroke therapy

T he Alberta Stroke Program Early CT Score merges the ability of quantifying and describing the topography of brain tissue damage produced by acute ischemic stroke in a semiquantitative way. ${ }^{1}$ ASPECTS on noncontrast CT is widely used for the assessment of early ischemic changes, and its prognostic value has already been established, ${ }^{2}$ though with poor NCCT sensitivity. ${ }^{3}$ Re-

Received October 14, 2016; accepted after revision March 24, 2017.

From the Department of Neuroscience (F.S., C.M., S.P., M.D., B.R., M.P., F.A., P.S., G.K.), Comprehensive Stroke Center, and Interventional Radiology and Neuroradiology (M.S., S.F., R.G., R.F.), Department of Diagnostic Imaging, University Hospital of Tor Vergata, Rome, Italy; Santa Lucia Foundation (C.M., G.K.), Rome, Italy; and Department of Medicine and Neurology (F.A.), Royal Melbourne Hospital, University of Melbourne, Parkville, Australia.

This work was supported by a grant from the Italian Ministry of Health (RF-2013-02358679).

Ethics approval for this study was obtained from the Tor Vergata Policlinic ethics committee.

Please address correspondence to Fabrizio Sallustio, MD, University Hospital of Tor Vergata, Viale Oxford 81, 00133, Rome, Italy; e-mail: fsall75@gmail.com

-- Indicates open access to non-subscribers at www.ajnr.org

http://dx.doi.org/10.3174/ajnr.A5264 cent randomized controlled trials on endovascular stroke therapy (ET) have been based on strict inclusion criteria, leading to treatment of only those patients with high CT ASPECTS indicating smaller infarct burden. ${ }^{4-6}$

Many attempts have been made to understand which patients are likely to undergo futile reperfusion. ${ }^{7}$ For instance, it has been recently demonstrated that patients with poor collaterals and longer time to reperfusion do not achieve good outcomes after ET. ${ }^{8}$ Thus, a careful patient selection for ET should be desirable and should be based on a multimodal neuroimaging approach in addition to onset time and stroke severity. Although not as commonly available as NCCT in the acute ischemic stroke setting, CT angiography is useful for confirmation of vessel occlusion in candidates for ET, and hypodensity on CTA source images (CTA-SI) has been shown to reliably correlate with ischemic lesion volume on diffusion-weighted imaging ${ }^{9}$ and final infarct size. ${ }^{10}$ The superiority of CTA-SI on NCCT in the detection of infarcted areas has been demonstrated for readers of all levels of experience. ${ }^{11}$ Few data exist on the value of CTA-SI ASPECTS in patients un- 
dergoing ET for acute ischemic stroke, ${ }^{12,13}$ and this lack of data may explain why only ASPECTS NCCT is currently considered in the guidelines for eligibility for ET. Our purpose was to investigate whether CTA-SI ASPECTS correlate better than NCCT ASPECTS with clinical and radiologic outcome measures in patients with acute ischemic stroke undergoing ET.

\section{MATERIALS AND METHODS}

A retrospective analysis of patients identified from a prospective registry at a comprehensive stroke center (University Hospital of Tor Vergata, Rome, Italy) was performed. Patients with anterior circulation acute ischemic stroke secondary to intracranial proximal arterial occlusion (M1 MCA, M2 MCA, distal internal carotid artery, and proximal ICA plus intracranial proximal arterial occlusion) admitted within 6 hours of symptom onset were included. Due to the study period (between 2009 and 2015) before the publication of recent endovascular stroke trials, ${ }^{4-6}$ no exclusion criteria other than the time from symptom onset were adopted. Patients presenting within 4.5 hours of symptom onset were treated with intravenous thrombolysis, which was continued in the angiographic suite during the endovascular procedure. $\mathrm{Pa}$ tients presenting beyond the time window for intravenous thrombolysis or with major contraindications to intravenous thrombolysis (ie, warfarin therapy with an international normalized ratio of 1.7, recent major surgery, or a history of hemorrhage/hematoma) underwent stand-alone thrombectomy. Demographics, vascular risk factors, and baseline and 24-hour NIHSS scores were reported. The modified Rankin Scale was adopted for outcome analysis, and an mRS of $\leq 2$ at 3 months was considered a favorable outcome. The study was approved by the Tor Vergata Policlinic Ethical Committee, and informed consent was obtained from all patients or their relatives.

\section{Image Acquisition}

The NCCT and CTA were acquired with a standardized protocol. Axial CT was performed on a multisection scanner (Light Speed VCT; GE Healthcare, Milwaukee, Wisconsin) by using $120 \mathrm{kV}$ and $170 \mathrm{mAs}$ with a $5-\mathrm{mm}$ section thickness. Continuous axial sections parallel to the orbitomeatal line were obtained from the skull base to the vertex. CTA was performed with a 64-detector row scanner. Acquisitions were obtained after single-bolus intravenous contrast injection of $90-120 \mathrm{~mL}$ of nonionic contrast media into an antecubital vein at $3-5 \mathrm{~mL} / \mathrm{s}$. Imaging was autotriggered by the appearance of contrast media in the ascending aorta. Standard coverage included the area from the arch to the vertex. Source images were reconstructed at a $1.25-\mathrm{mm}$ thickness in the axial planes at half-thickness intervals. NCCT or DWI was performed between 1 and 7 days after stroke onset and used for follow-up ASPECTS.

\section{Image Processing}

NCCT, CTA-SI, and MR imaging (DWI sequences and apparent diffusion coefficient map) scans were independently screened for ASPECTS by 1 neuroradiologist (M.S.) and 1 stroke neurologist (G.K.) who were blinded to the patients' symptoms but aware of acute nonlacunar stroke. The readers performed their evaluation at different time periods to make their assessment blinded as much as possible. Adequate window and optimal level settings were adopted to maximize the contrast produced by attenuation differences between normal and ischemic tissue. Our ASPECTS reading includes evaluation of all axial sections (www.aspectsinstroke.com), and as in previous studies, we excluded isolated cortical swelling from the score. ${ }^{14}$ In case of a discrepancy between readers, a third neuroradiologist (R.G.) was involved to achieve a consensus. The interrater reliability was 0.71 for NCCT and 0.75 for CTA-SI, indicating a good interrater agreement for both methods.

\section{Statistical Analysis}

The analysis was performed by using STATA/IC, Version 13 (StataCorp, College Station, Texas) and GraphPad Prism software, Version 6.00 (GraphPad Software, San Diego, California). Continuous variables are summarized as mean $\pm \mathrm{SD}$ or median with interquartile range. Categoric variables are expressed as percentages. To determine differences between the 2 groups, we used a Student $t$ test or Mann-Whitney $U$ test for continuous variables. Interrater agreement was estimated with the $\kappa$ statistic. Comparison of frequencies among ASPECTS groups was performed with the Fisher exact test after dichotomization into poor and good ASPECTS. Univariate associations between baseline and follow-up ASPECTS were investigated with the Spearman $\rho$ analysis, with confidence limits calculated by means of the Fisher $z$-transformation. Bubble plots were used to graphically display correlation analyses, and the area of the bubble has to be read as proportional to the number of observations at each point. A nonparametric receiver operating characteristic curve analysis and the area under the curve were used to assess the ability of NCCT and CTA-SI ASPECTS to identify patients with favorable outcomes ( $\mathrm{mRS} \leq 2)$.

We then calculated the statistical significance of the difference between the area under the curves using the method of DeLong. ${ }^{15}$ For both NCCT and CTA-SI ASPECTS, a receiver operating characteristic curve was used to identify the best cutoff point with which to maximize the sensitivity and specificity for discriminating patients with favorable outcomes. A backward and forward stepwise logistic regression analysis was finally performed to determine the independent predictors of good outcome $(\mathrm{mRS} \leq 2)$, including NCCT and CTA-SI ASPECTS as well as all other variables with a significant association in univariate analysis, to weigh for potentially confounding factors. Odds ratios with standard errors and 95\% confidence intervals were provided. A $P$ value $<.05$ was considered statistically significant.

\section{RESULTS}

Of 167 patients with anterior circulation stroke, 124 had complete CT, CTA, and clinical data and were included in the analysis. Baseline characteristics are summarized in Table 1. Single-artery occlusion (ICA, anterior cerebral artery, and middle cerebral artery) was diagnosed in $55.6 \%$, whereas tandem lesions occurred in $44.3 \%$ of patients. Correlation analysis showed that baseline CTA-SI ASPECTS correlated better with follow-up ASPECTS $(r=0.76 ; 95 \%$ CI, 0.67-0.83; $P<.001)$ than baseline NCCT ASPECTS $(r=0.51 ; 95 \%$ CI, $0.36-63 ; P<.001 ; P$ for comparison 
of the 2 coefficients $<.001$ ) (Fig 1 ). Furthermore, the ability to identify patients with good outcome $(\mathrm{mRS} \leq 2)$, revealed by receiver operating characteristic curve analysis, was significantly higher for CTA-SI ASPECTS with respect to NCCT ASPECTS (CTA-SI area under the curve, 0.83; 95\% CI, 0.76-0.91; NCCT area under the curve, $0.67 ; 95 \% \mathrm{CI}, 0.58-0.77 ; P<.001)$. According to each receiver operating characteristic curve, we determined cutoff values (Table 2), on the basis of which we defined a good CTA-SI ASPECTS as $\geq 5$ and a good NCCT ASPECTS as $\geq 8$. Most interesting, a median baseline NCCT ASPECTS of 9 resulted from a recent meta-analysis of 5 endovascular stroke trials. ${ }^{16}$ No statistical difference was found in onset-to-imaging acquisition time between good and poor ASPECTS groups (good CTA-SI, $135 \pm 56$ minutes versus poor CTA-SI, $137 \pm 52$ minutes; $P>.05$; good NCCT, $129 \pm 54$ minutes versus poor NCCT, $131 \pm 54$ minutes; $P>.05)$.

Factors predicting favorable outcome $(\mathrm{mRS} \leq 2, n=48)$ in univariate analysis were both good NCCT ASPECTS and good CTA-SI ASPECTS, as well as age, baseline NIHSS, time to recanalization, and good recanalization (TICI $\geq 2 \mathrm{~b}$ ) (Table 3 ). To find the best outcome predictors, we finally constructed backward and forward stepwise regression analyses, including all variables significantly associated with favorable outcome in the univariate

Table 1: Patient characteristics

\begin{tabular}{lc}
\hline \multicolumn{1}{c}{ Variable } & Value \\
\hline Age (yr) & $68 \pm 13.2$ \\
Sex (male) & $52.4 \%$ \\
Hypertension & $79.8 \%$ \\
Diabetes & $16.1 \%$ \\
Atrial fibrillation & $32.2 \%$ \\
Smoking & $19.3 \%$ \\
Intravenous thrombolysis & $60.4 \%$ \\
Baseline NIHSS & $19(\mathrm{IQR}, 16-21)$ \\
NCCT ASPECTS & $8(\mathrm{IQR}, 7-10)$ \\
CTA-SI ASPECTS & $4(\mathrm{IQR}, 2-6)$ \\
Follow-up ASPECTS & $3(\mathrm{IQR}, 1-5)$ \\
\hline
\end{tabular}

Note:- IQR indicates interquartile range.

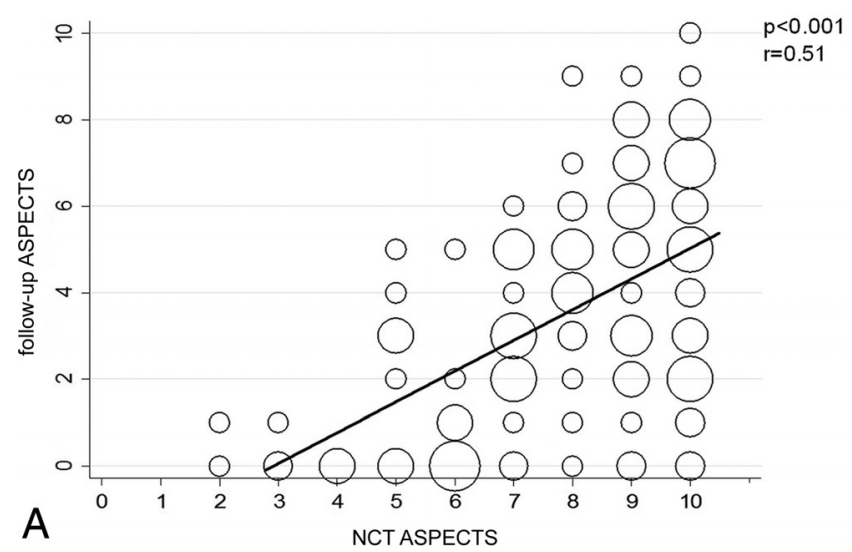

analysis. Both the backward and forward procedures showed that good CTA-SI ASPECTS, age, good recanalization, and time to recanalization remained independent predictors of good clinical outcome, indicating CTA-SI ASPECTS as a better predictor of functional outcome than NCCT ASPECTS (Table 4).

\section{DISCUSSION}

ET seems a safe and effective adjuvant treatment strategy for patients with acute ischemic stroke secondary to large intracranial vessel occlusion in the anterior circulation, ${ }^{4,17}$ and many attempts have been made in the recent past to better select those patients who can reliably benefit from ET. ${ }^{7}$ One such effort has been in recent randomized endovascular stroke trials that included only patients with small infarct size as defined by an ASPECTS of $>6-7$ on NCCT. ${ }^{5,6}$ Among these trials, the Multicenter Randomized Clinical trial of Endovascular treatment for Acute ischemic stroke in the Netherlands (MR CLEAN) was the only one to include patients on the basis of time from symptom onset and confirmation of occlusion on neuroimaging. ${ }^{4}$ Nevertheless, a recent meta-analysis of these trials showed a median baseline CT ASPECTS of 9. Consequently, from all these trials, we have learned that ET works, but we do not know yet whether this kind of reperfusion therapy would be safe and effective in patients with lower ASPECTS.

Regarding these findings, a recent subgroup analysis from the MR CLEAN data showed that patients with NCCT ASPECTS of $5-7$, not only those with NCCT ASPECTS of 8-10, may also benefit from ET. This study could not provide further information on patients with NCCT ASPECTS of $0-4$ because of a paucity of data. ${ }^{18}$ Therefore, there are not thorough data concerning the effects of ET in patients with larger infarct burdens, and we only know that those with lower ASPECTS could be unsuitable for $\mathrm{ET}^{19}$ but cannot be excluded from treatment. A recent study of 249 patients showed a rate of good outcome of $5 \%$ in the CT ASPECTS group of $0-4$ and of $38.5 \%$ in the CT ASPECTS group of $5-8,{ }^{7}$ suggesting a chance for ET in patients

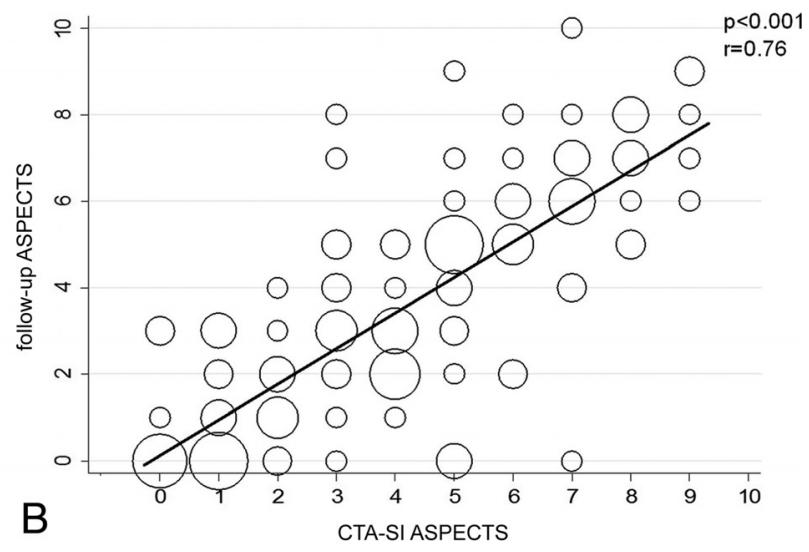

FIG 1. Correlation of ASPECTS on NCCT $(A)$ and CTA-SI (B) with follow-up ASPECTS. The area of the bubble is proportional to the number of patient observations at that data point.

Table 2: ROC curve analysis-CTA-SI ASPECTS and NCCT ASPECTS cutoff values indicating patients with good clinical outcomes

\begin{tabular}{lccccc}
\hline & AUC $(95 \% \mathrm{Cl})$ & Best Cutoff Value & Sensitivity & Specificity & Correct Classification \\
\hline CTA-SI ASPECTS & $0.83(0.76-0.91)$ & 5 & $60.42 \%$ & $88.16 \%$ & $77.42 \%$ \\
NCCT ASPECTS & $0.67(0.58-0.77)$ & 8 & $64.58 \%$ & $61.84 \%$ & $62.90 \%$ \\
\hline
\end{tabular}

Note:-AUC indicates area under curve; ROC, receiver operating characteristic. 
Table 3: Univariate analysis-variables associated with good clinical outcome

\begin{tabular}{lccc}
\hline \multicolumn{1}{c}{ Variable } & mRS 0-2 ( $\mathbf{n = 4 8 )}$ & mRS 3-6 (n= 76) & $\boldsymbol{P}$ \\
\hline Age (yr) & $63.3 \pm 15.3$ & $70.3 \pm 11.0$ & .004 \\
Sex (male) & $52 \%$ & $52 \%$ & 1.0 \\
Baseline NIHSS & $17.6 \pm 3.1(\mathrm{IQR}, 11-23)$ & $19.3 \pm 3.5(\mathrm{IQR}, 9-25)$ & .009 \\
Good NCCT ASPECTS & $81.2 \%$ & $50 \%$ & .001 \\
Good CTA-SI ASPECTS & $79.2 \%$ & $26.3 \%$ & $<.001$ \\
Onset to recanalization (min) & $291.9 \pm 86.3(\mathrm{IQR}, 140-635)$ & $322.5 \pm 70.3(\mathrm{IQR}, 155-505)$ & .03 \\
Hypertension & $70.8 \%$ & $85.5 \%$ & .07 \\
Diabetes & $14.6 \%$ & $17.3 \%$ & .80 \\
Atrial fibrillation & $33.3 \%$ & $31.6 \%$ & .84 \\
Smoking & $18.7 \%$ & $19.7 \%$ & 1.0 \\
Intravenous thrombolysis & $93.7 \%$ & $90.8 \%$ & .74 \\
sICH & $23.4 \%$ & $14.7 \%$ & .23 \\
TICI 2b-3 & $79.2 \%$ & $56.6 \%$ & .01 \\
\hline
\end{tabular}

Note:- $s \mathrm{ICH}$ indicates symptomatic intracranial hemorrhage.

Table 4: Multivariable regression model—best predictors of good clinical outcome

\begin{tabular}{lcccc}
\hline & OR & SE & $95 \%$ Cl & $P$ \\
\hline Good CTA-SI ASPECTS & 15.61 & 8.24 & $5.54-43.97$ & $<.001$ \\
Age & 0.95 & 0.02 & $0.91-0.99$ & .011 \\
TICl 2b-3 & 3.12 & 1.70 & $1.07-9.09$ & .037 \\
Onset to recanalization (min) & 0.99 & 0.01 & $0.98-0.99$ & .009
\end{tabular}

Note:-SE indicates standard error.

with larger infarct burden. Further scientific effort is warranted to identify any markers that could better predict outcome and give useful information for the decision-making process.

Thus, CTA can depict the area of ischemia (though not necessarily infarcted) much better than NCCT, especially when analyzing parenchymal CTA-SI. ${ }^{12}$ Hypodensity on CTA-SI provides greater demarcation between normal and abnormal tissue, and this finding could be explained by the ability of CTA to detect alterations in cerebral blood volume, as opposed to cytotoxic edema on NCCT, with a threshold insufficient to produce NCCT changes. ${ }^{11}$ In a small previous study, CTA-SI was shown to be more sensitive in the early detection of irreversible ischemia and more accurate in the prediction of final infarct size. ${ }^{13}$ Our results confirm those from Bhatia et al, ${ }^{20}$ suggesting CTA-SI ASPECTS prior to ET as a better predictor of final infarction. In our study, we also compared CTA-SI ASPECTS and NCCT ASPECTS to evaluate whether the former can improve prediction of clinical outcome. We used receiver operating characteristic curve analysis and found that baseline CTA-SI ASPECTS compared with NCCT ASPECTS can better identify patients with functional independence at 3 months.

Moreover, our study, different from the study of Bhatia et al, ${ }^{20}$ also analyzed reperfusion data. In particular, we found that good reperfusion was a predictor of good outcome in univariate analysis and remains a significant and independent predictor of good outcome in a multivariable regression model together with age, time to reperfusion, and CTA-SI ASPECTS, but not NCCT ASPECTS. In line with this finding, good reperfusion has been recently demonstrated to improve the rate of good outcome irrespective of ASPECTS. $^{21}$

\section{Limitations}

Our study has several limitations primarily due to the retrospective methodology of analysis. Moreover, the sample size

\section{ACKNOWLEDGMENTS} MD) for data collection. paid to the institution

\section{REFERENCES} Medline CrossRef Medline was relatively small and may limit the reliability of results. Larger multicenter studies are needed to conclusively demonstrate the utility of CTASI in clinical decision-making.

\section{CONCLUSIONS}

Our study shows that CTA-SI ASPECTS can predict final infarct size and outcome better than NCCT ASPECTS. This finding suggests that CTA-SI, rather than NCCT, should be considered a main step of the decision-making process in patients with acute ischemic stroke.

We thank all stroke neurologists of the Comprehensive Stroke Center at the University of Tor Vergata (Angela Giordano, MD, Vittoria Carla D'Agostino, $\mathrm{PhD}$, Barbara Rizzato, $\mathrm{PhD}$, Simone Napolitano, MD, Domenico Samà, $\mathrm{PhD}$ ) and interventional radiologists of the Department of Diagnostic Imaging at the University of Tor Vergata (Daniel Konda, MD, Enrico Pampana,

Disclosures: Fabrizio Sallustio-RELATED: Grant: Italian Ministry of Health (RF-201302358679)*. Caterina Motta—RELATED: Grant: Italian Ministry of Health (RF-201302358679)*. Marina Diomedi-UNRELATED: Payment for Lectures Including Service on Speakers Bureaus: Italian Society of Neurology (Symposium of Bristol MyersSquibb and Pfizer), Comments: $€ 1000$ for a lecture. Paolo Stanzione-UNRELATED: Board Membership: Union Chimique Belge (UCB), Sandoz, Comments: Advisory Board; Grants/Grants Pending: UCB, Boehringer, Comments: unrestricted grants for sleep disturbances in Parkinson disease, unrestricted grant for a Masters Degree in Neurovascular Disease*; Payment for Lectures Including Service on Speakers Bureaus: $\mathrm{UCB}$, Chiesi, Comments: Continuous Education in Medicine in Italy. ${ }^{*}$ Money

1. Barber PA, Demchuk AM, Zhang J, et al; ASPECTS Study Group. Validity and reliability of a quantitative computed tomography score in predicting outcome of hyperacute stroke before thrombolytic therapy. Lancet 2000;355:1670-74 CrossRef Medline

2. Dzialowski I, Hill MD, Coutts SB, et al. Extent of early ischemic changes on computed tomography (CT) before thrombolysis: prognostic value of the Alberta Stroke Program Early CT Score in ECASS II. Stroke 2006;37:973-78 CrossRef Medline

3. Menon BK, Puetz V, Kochar P, et al. ASPECTS and other neuroimaging scores in the triage and prediction of outcome in acute stroke patients. Neuroimaging Clin North Am 2011;21:407-23 CrossRef

4. Berkhemer OA, Fransen PS, Beumer D, et al. A randomized trial of intraarterial treatment for acute ischemic stroke. N Engl J Med 2015; 372:11-20 CrossRef Medline

5. Goyal M, Demchuk AM, Menon BK, et al. Randomized assessment of rapid endovascular treatment of ischemic stroke. $N$ Engl J Med 2015;372:1019-30 CrossRef Medline

6. Jovin TG, Chamorro A, Cobo E, et al. Thrombectomy within $\mathbf{8}$ hours after symptom onset in ischemic stroke. N Engl J Med 2015;372: 2296-306 CrossRef Medline

7. Yoo AJ, Zaidat OO, Chaudhry ZA, et al. Impact of pretreatment noncontrast CT Alberta Stroke Program Early CT score on clinical outcome after intra-arterial stroke therapy. Stroke 2014;45:746-51

8. Sallustio F, Motta C, Pizzuto S, et al. CT angiography-based collat- 
eral flow and time to reperfusion are strong predictors of outcome in endovascular treatment of patients with stroke. J Neurointerv Surg 2016 Sep 23. [Epub ahead of print] CrossRef Medline

9. Schramm P, Schellinger PD, Fiebach JB, et al. Comparison of CT and $\mathrm{CT}$ angiography source images with diffusion-weighted imaging in patients with acute stroke within 6 hours after onset. Stroke 2002; 33:2426-32 CrossRef Medline

10. Lev MH, Segal AZ, Farkas J, et al. Utility of perfusion-weighted CT imaging in acute middle cerebral artery stroke treated with intraarterial thrombolysis: prediction of final infarct volume and clinical outcome. Stroke 2001;32:2021-28 CrossRef Medline

11. Aviv RI, Shelef I, Malam S, et al. Early stroke detection and extent: impact of experience and the role of computed tomography angiography source images. Clin Radiol 2007;62:447-52 CrossRef Medline

12. Nabavi DG, Kloska SP, Nam EM, et al. MOSAIC: Multimodal Stroke Assessment Using Computed Tomography-novel diagnostic approach for the prediction of infarction size and clinical outcome. Stroke 2002;33:2819-26 CrossRef Medline

13. Camargo EC, Furie KL, Singhal AB, et al. Acute brain infarct: detection and delineation with $\mathrm{CT}$ angiographic source images versus nonenhanced CT scans. Radiology 2007;244:541-48 CrossRef Medline

14. Puetz V, Dzialowski I, Hill MD, et al. The Alberta Stroke Program Early CT Score in clinical practice: what have we learned? Int J Stroke 2009;4:354-64 CrossRef Medline

15. DeLong ER, DeLong DM, Clarke-Pearson DL. Comparing the areas under two or more correlated receiver operating characteristic curves: a nonparametric approach. Biometrics 1988;44:837-45
16. Goyal M, Menon BK, van Zwam WH, et al; HERMES collaborators. Endovascular thrombectomy after large-vessel ischaemic stroke: a meta-analysis of individual patient data from five randomised trials. Lancet 2016;387:1723-31 CrossRef Medline

17. Sallustio F, Koch G, Di Legge S, et al. Intra-arterial thrombectomy versus standard intravenous thrombolysis in patients with anterior circulation stroke caused by intracranial occlusions: a single-center experience. J Stroke Cerebrovasc Dis 2013;22:e323-31 CrossRef Medline

18. Yoo AJ, Berkhemer OA, Fransen PS, et al; MR CLEAN investigators. Effect of baseline Alberta Stroke Program Early CT Score on safety and efficacy of intra-arterial treatment: a subgroup analysis of a randomised phase 3 trial (MR CLEAN). Lancet Neurol 2016;15: 685-94 CrossRef Medline

19. Wahlgren N, Moreira T, Michel P, et al; ESO-KSU, ESO, ESMINT, ESNR and EAN. Mechanical thrombectomy in acute ischemic stroke: consensus statement by ESO-Karolinska Stroke Update 2014/2015, supported by ESO, ESMINT, ESNR and EAN. Int J Stroke 2016;11:134-47 CrossRef Medline

20. Bhatia R, Bal SS, Shobha N, et al; Calgary CTA Group. CT angiographic source images predict outcome and final infarct volume better than noncontrast $\mathrm{CT}$ in proximal vascular occlusions. Stroke 2011;42:1575-80 CrossRef Medline

21. Noorian AR, Rangaraju S, Sun $\mathrm{CH}$, et al. Endovascular therapy in strokes with ASPECTS 5-7 may result in smaller infarcts and better outcomes as compared to medical treatment alone. Interv Neurol 2015;4:30-37 CrossRef Medline 